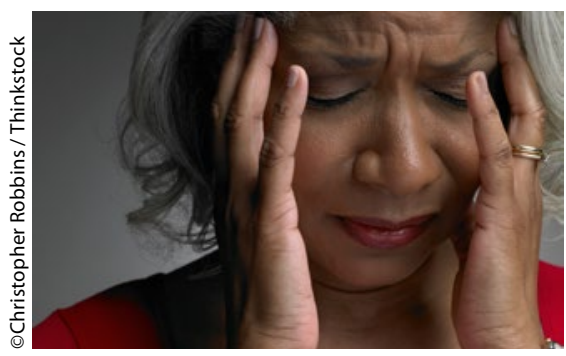

Christoph Diener von der Universitätsklinik Essen und Professor David Dodick von der Mayo Clinic in Phoenix, USA, verweisen auf eine 2015 publizierte Metaanalyse zu 132 placebokontrollierten Studien. Sie ergab für die bislang am häufigsten verwendeten Prophylaktika nur eine Reduktion von 0,5 bis 1,7 Tagen im Vergleich zu Placebo. Vorteile der CGRP-Antagonisten seien zudem die gute Verträglichkeit und die einmal monatliche Anwendung per Injektion. Dadurch gebe es kaum Probleme mit der Compliance, schreiben die Neurologen.

mut

2015 Round-up. The Lancet Neurology 2016; 15 (1): $2-21$

\title{
Bewegungsstörungen - Auftrieb für Darm- und Prionhypothese
}

C chon lange hält sich der Verdacht, $\checkmark$ dass einige Bewegungsstörungen durch fehlgefaltete Proteine verursacht werden, die sich wie Prionen im Gehirn ausbreiten und dabei ihresgleichen in eine pathogene Form zwingen. In Tierexperimenten konnte der Prionenentdecker Professor Stanley Prusiner Mäuse mit Hirngewebe von Patienten infizie- ren, die an Multisystematrophie (MSA) erkrankt waren. Es reichte eine intramuskuläre Injektion, damit die Tiere ebenfalls eine MSA-ähnliche Krankheit entwickelten. Prusiner vermutet, dass auch Morbus Parkinson und Lewy-Körperchen-Demenz durch prionähnliche Alpha-Synuclein-Aggregate entstehen was noch in entsprechenden Tiermodel- len zu zeigen wäre. Für Dr. Nobutaka Hatori von der Universität in Tokyo sind auch Ergebnisse einer dänischen Registerstudie zu Patienten mit Vagotomie interessant: Solche Patienten erkranken offenbar seltener an Parkinson. Dies lässt sich als weiterer Hinweis deuten, dass die Krankheit im enterischen Nervensystem entsteht und sich von dort aus ins Gehirn ausbreitet.

mut

2015 Round-up. The Lancet Neurology 2016; 15 (1): 2-21

\section{Schlafstörungen - CPAP gegen Demenz}

$\mathrm{D}$ ie nächtliche Beatmung ist bei Schlafapnoe offenbar die beste Demenzprävention. So lässt sich zumindest eine Auswertung der Alzheimer's Disease Neuroimaging Initiative (ADNI) interpretieren. Danach waren Patienten mit Schlafapnoe bei der MCI (mild cognitive impairment)-Diagnose im Schnitt zehn und bei der Alzheimer-Diagnose fünf Jahre jünger als solche ohne nächtliche Atemprobleme. Keinen Nachteil hatten jedoch Schlafapnoepatienten, die CPAP nutzten. Möglicherweise lässt sich eine MCI also um bis zu zehn Jahre verzögern.

Dr. Cathy Goldstein und Dr. Ronald Chervin von der Universität in Ann Arbor, USA, weisen auf eine weitere interessante Studie hin: Probanden mit weniger als sechs Stunden Schlaf zogen sich bei Rhinovirenexposition viermal häufiger eine Erkältung zu als solche, die ausschlafen konnten.

mut

2015 Round-up. The Lancet Neurology 2016; 15 (1): $2-21$

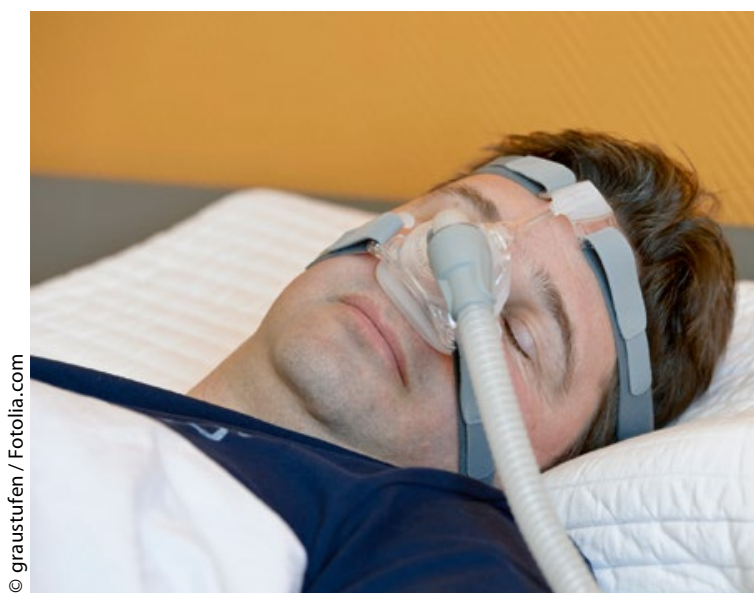

\section{Epilepsie - besseres Therapiemanagement}

omogramme werden in der Onkologie schon lange zur Abschätzung der individuellen Prognose eingesetzt. 2015 wurde nun ein ähnliches Verfahren an der Cleveland Clinic in den USA für die Epilepsiechirurgie entwickelt. Ärzte sollen damit anhand der jeweiligen $\mathrm{Pa}$ tientencharakteristika abschätzen können, wie hoch die Wahrscheinlichkeit ist, nach der Operation über zwei und fünf Jahre anfallsfrei zu bleiben. Das Verfahren müsse nun aber prospektiv evaluiert werden, berichtet Dr. Elinor Ben-Menachem von der Universität in Göteborg, Schweden. Für wichtig hält die Neurologin auch neue Daten zu den Risiken von Valproat in der Schwangerschaft. Danach sind Dosierungen von weniger als
$800 \mathrm{mg} /$ Tag nicht mit kognitiven Einschränkungen beim Nachwuchs verbunden. Dies sei eine wichtige Information, da Valproat bei manchen Epilepsieformen zu den wirksamsten Substanzen zähle und nicht immer durch andere Antikonvulsiva ersetzt werden könne.

mut

2015 Round-up. The Lancet Neurology 2016; 15 (1): $2-21$ 